\title{
Article \\ Comparison of Controller-Based Locomotion Techniques for Visual Observation in Virtual Reality
}

\author{
Jussi Rantala*D, Jari Kangas ${ }^{D}$, Olli Koskinen, Tomi Nukarinen and Roope Raisamo \\ TAUCHI Research Center, Computing Sciences, Tampere University, 33100 Tampere, Finland; \\ jari.a.kangas@tuni.fi (J.K.); olli.koskinen@staff.uta.fi (O.K.); tomi.nukarinen@tuni.fi (T.N.); \\ roope.raisamo@tuni.fi (R.R.) \\ * Correspondence: jussi.rantala@tuni.fi
}

Citation: Rantala, J.; Kangas, J.;

Koskinen, O.; Nukarinen, T.; Raisamo,

R. Comparison of Controller-Based

Locomotion Techniques for Visual Observation in Virtual Reality.

Multimodal Technol. Interact. 2021, 5,

31. https://doi.org/10.3390/

mti5070031

Academic Editor: Gerd Bruder

Received: 26 April 2021

Accepted: 18 June 2021

Published: 23 June 2021

Publisher's Note: MDPI stays neutral with regard to jurisdictional claims in published maps and institutional affiliations.

Copyright: (c) 2021 by the authors. Licensee MDPI, Basel, Switzerland. This article is an open access article distributed under the terms and conditions of the Creative Commons Attribution (CC BY) license (https:// creativecommons.org/licenses/by/ $4.0 /)$.
Abstract: Many virtual reality (VR) applications use teleport for locomotion. The non-continuous locomotion of teleport is suited for VR controllers and can minimize simulator sickness, but it can also reduce spatial awareness compared to continuous locomotion. Our aim was to create continuous, controller-based locomotion techniques that would support spatial awareness. We compared the new techniques, slider and grab, with teleport in a task where participants counted small visual targets in a VR environment. Task performance was assessed by asking participants to report how many visual targets they found. The results showed that slider and grab were significantly faster to use than teleport, and they did not cause significantly more simulator sickness than teleport. Moreover, the continuous techniques provided better spatial awareness than teleport.

Keywords: virtual reality; locomotion methods; locomotion techniques; visual observation

\section{Introduction}

Virtual travel, or locomotion, is a fundamental part of virtual reality (VR) because it is one of the most frequently performed tasks [1,2]. Locomotion is typically needed in all VR applications to move from one location to another. Essentially, it consists of actions that change the user's orientation and viewpoint [1]. Researchers have proposed a range of different locomotion techniques since the early days of VR development. This research has gathered more pace after the VR revival, which was started by the introduction of Oculus Rift DK 1 in 2013. The sophisticated head and controller position tracking in Oculus Rift and other related headsets has facilitated locomotion technique development.

A literature review [3] of recent VR locomotion papers identified 11 main locomotion technique types: real walking [4-6], walking-in-place [7-9], controller/joystick [10-12], gesture-based [13-15], teleport [6,7,12,14-19], redirected walking [20-23], arm swinging [4,24], reorientation [25], head-directed [26,27], human joystick [8,28], and chair-based [27]. A database of over 100 locomotion techniques [2] can be used for the comparison of techniques. Except for teleport, all the other locomotion techniques listed above are based on continuous locomotion where the visual environment is updated in a smooth and uninterrupted manner. This resembles human movement in a physical space. Teleport, on the contrary, uses non-continuous locomotion where the user moves to the desired virtual target location instantaneously.

The contrast between research interest in continuous and non-continuous locomotion is notable because the less studied non-continuous teleport is the default locomotion technique in numerous VR applications. While teleport is widely used and appropriate, especially for quick travel between locations [29], it is not an ideal technique in all situations. Teleport is less suitable for observing the travel route [29], and it can also cause disorientation and reduce spatial awareness [30,31] unless optimized for retaining some optical flow cues [32]. 
One main reason for the popularity of teleport is that it does not induce simulator sickness, also called cybersickness or VR sickness, as easily as continuous locomotion where a mismatch in sensory information between the visual and other senses can lead to a headache, blurred vision, dizziness, disorientation, or nausea [1]. In practice, our eyes inform our brain that we are moving, but the vestibular and proprioceptive systems do not receive corresponding motion cues. Continuous locomotion techniques that provide these motion cues are less susceptible to inducing simulator sickness. For example, real walking $[4,5,33]$, redirected walking $[20,21,34]$, and reorientation [25] reduce the mismatch in sensory information because they map physical walking or walking-like gestures to locomotion in VR. However, walking-based locomotion techniques are not always feasible. For instance, arranging a large, tracked space can be impossible, the user may prefer to remain stationary, or the user is unable to walk because of a disability [11]. In these scenarios, a controller-based, continuous locomotion technique could be the best option.

The challenge lies in finding a continuous locomotion technique that does not induce simulator sickness despite the stationary user. In two studies [29,35], users initiated continuous movement in VR by pressing a button on the HTC Vive controller. The results of user studies showed that this induced significantly more simulator sickness than the use of non-continuous teleport with the same controller. One alternative to address the challenge is to use dynamic field-of-view modification [11] or rest frames [36]. Both methods of visual scene modification were shown to be effective in reducing simulator sickness because participants stayed in VR longer compared to conditions with no visual modifications. Another alternative could be to modify the controller-based locomotion technique itself so that it reduces the mismatch between vision and other senses. The latter approach is studied in this paper.

The current aim was to find out whether it is possible to develop continuous, controllerbased locomotion techniques that would not induce significantly more simulator sickness than teleport. Such techniques could be valuable for observing the visual environment in VR during movement. For example, when performing building information modeling, the user may need to search for possible deviations in a virtual structure. Earlier work [29,30] suggests that teleport may not be optimal for a scenario such as this that requires good spatial awareness from the user. A possible reason for this could be the lack of visual and kinesthetic feedback of movement that can contribute to spatial knowledge [37]. Therefore, we also wanted to study whether continuous locomotion techniques offer a better sense of spatial awareness than teleport during movement in VR.

We conducted a user study with three locomotion techniques: slider, grab and teleport. Our goal in designing slider and grab was to support continuous movement, whereas teleport was based on non-continuous movement. We hypothesized that the continuous movement of slider and grab supports spatial awareness. All techniques utilized the HTC Vive headset and its controller. Participants used the techniques in a task where they moved inside a virtual house and simultaneously observed its structures. The techniques were compared by analyzing task completion times, the number of gestures used, and the number of errors. Additionally, we asked the participants to evaluate each technique with a Simulator Sickness Questionnaire (SSQ) [38], NASA Task Load Index (NASA-TLX) [39], and a custom task-related spatial awareness questionnaire.

\section{Methods}

\subsection{Participants}

Eighteen volunteers (7 males, 11 females) aged between 18 and 46 years (mean 25 years) took part in the experiment. All of them were either students or employees at a local university, and sixteen had used VR headsets at least once before. During the experiment, five participants wore the headset with glasses and one with contact lenses. Sixteen were right-handed. All participants signed an informed consent form before proceeding to the experiment. They were compensated for their effort with a movie ticket. 


\subsection{Experimental Setup}

The hardware used for setting up the experiment included an MSI GS63VR 7RF-218NE Stealth Pro laptop running Windows 10, HTC Vive headset, and one HTC Vive controller. The controller was held in the dominant hand. The participants sat on a non-swivel desk chair during the experiment. A seated condition was chosen to avoid falling. The chair was placed in the middle of a tracking space that was approximately $2.5 \times 2.0 \mathrm{~m}$ in size.

Once the participants were fitted with the headset and controller, they were introduced to a virtual house implemented with Unity (version 2018.1.0f2). The house comprised a hall and corridor with wooden floor and wall textures. The hall was used for rehearsing the use of the locomotion techniques. The corridor with dimensions of $24 \times 4 \times 2.6 \mathrm{~m}$ (length $\times$ width $\times$ height) was used in the visual observation task (see the left side of Figure 1). The left side wall of the corridor included small grey dots (diameter of 7 millimeters, see the right side of Figure 1) placed between two horizontal lines. The participants had to move slowly and focus on observing the wall to see the dots.
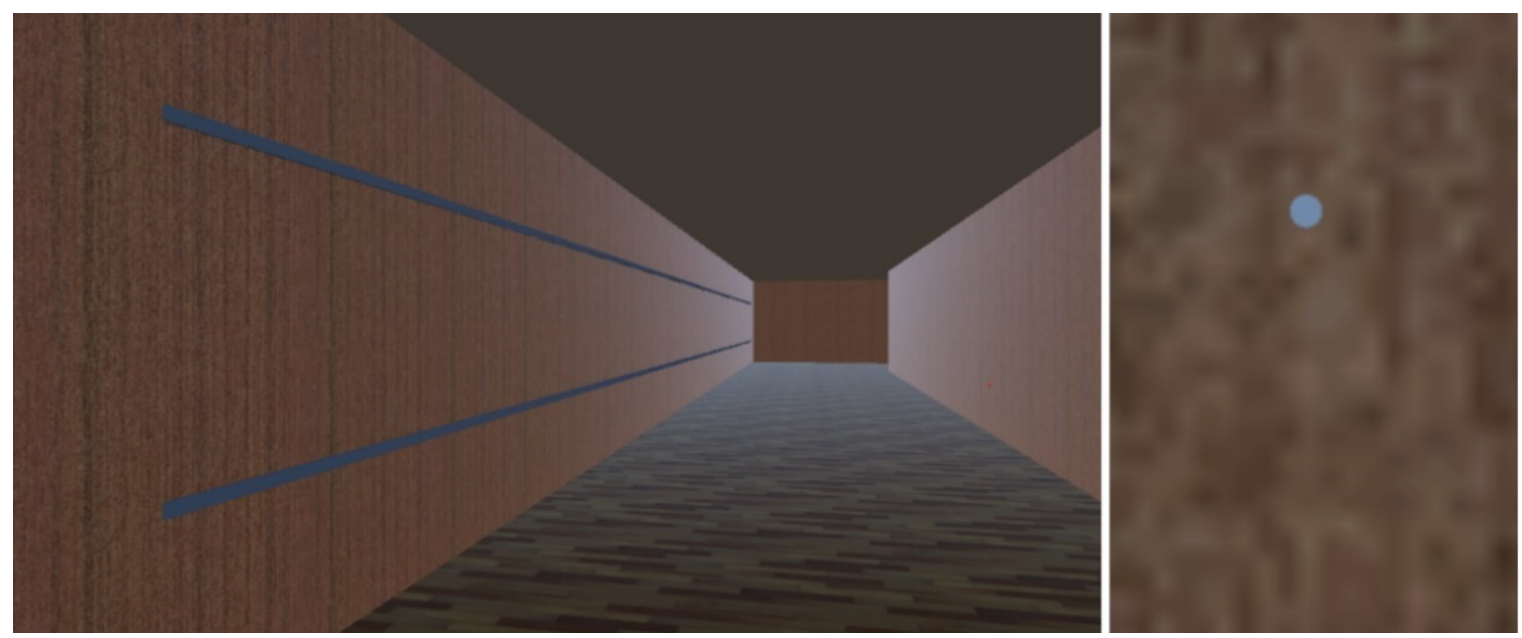

Figure 1. Grey dots to be counted were placed on the left side wall between two horizontal lines (on the left). An example dot on the wall (on the right).

\subsection{Locomotion Techniques}

We evaluated three locomotion techniques: teleport, slider, and grab. Teleport was chosen because it is one of the most common non-continuous locomotion techniques in current VR applications. Slider and grab were two alternative continuous locomotion techniques that were developed for the purpose of experimental comparison with teleport. With all techniques, the participants could change the direction they were facing in VR either by physically turning their heads or by using left-right rotation (yaw) with the controller while holding down its grip buttons. When using the controller, the direction in which the participant was facing was determined by the resulting pointing direction of the controller. The rest of the controller functionality was specific to each technique.

\subsubsection{Teleport}

Teleport was based on existing teleporting prefabs and scripts of SteamVR [40]. The default auditory and haptic feedback of teleport were disabled because the other two techniques did not have similar feedback. With teleport, the participant used a parabolic pointer to choose the desired location and pressed the touchpad of the controller to travel there (see the left side of Figure 2). Following the principle of teleport, the participant was transported to the new position instantly once the touchpad was released. The facing direction of the participant did not change in the new position. 


\subsubsection{Slider}

Slider was a novel technique developed by the authors. The closest earlier technique was "Ray-casting" [41] which did not support setting the speed of movement during travel. Slider was based on a metaphor where the participant would slide along a horizontal trajectory in a continuous motion. The trajectory was chosen by moving a virtual line that followed the pointing direction of the controller (see the middle of Figure 2). The desired trajectory was locked by pressing down the touchpad. The decision to use a locked trajectory was made after piloting where traversal with a non-locked trajectory was proven difficult.

To travel forward in VR, the participant moved the controller towards the body from the lock position. The green line was visible during travel to increase the predictability of the movement and, thus, possibly decrease the feeling of simulator sickness. The displacement of the controller $d_{c}$ was mapped to the speed of travel $s$ using a function

$$
s=m \times d_{c}^{2},
$$

where multiplier $m$ was set to 2.8 based on piloting. A quadratic function was chosen so that the participant could use small controller displacements for slow travel speeds, but also move faster with large controller displacements if needed.

Contrary to earlier work [42], the participant could look in different directions during travel by turning their heads. When the participant had reached their desired destination, they released the touchpad. To move backward, the controller was moved away from the body. The current implementation of slider did not support lateral movement.
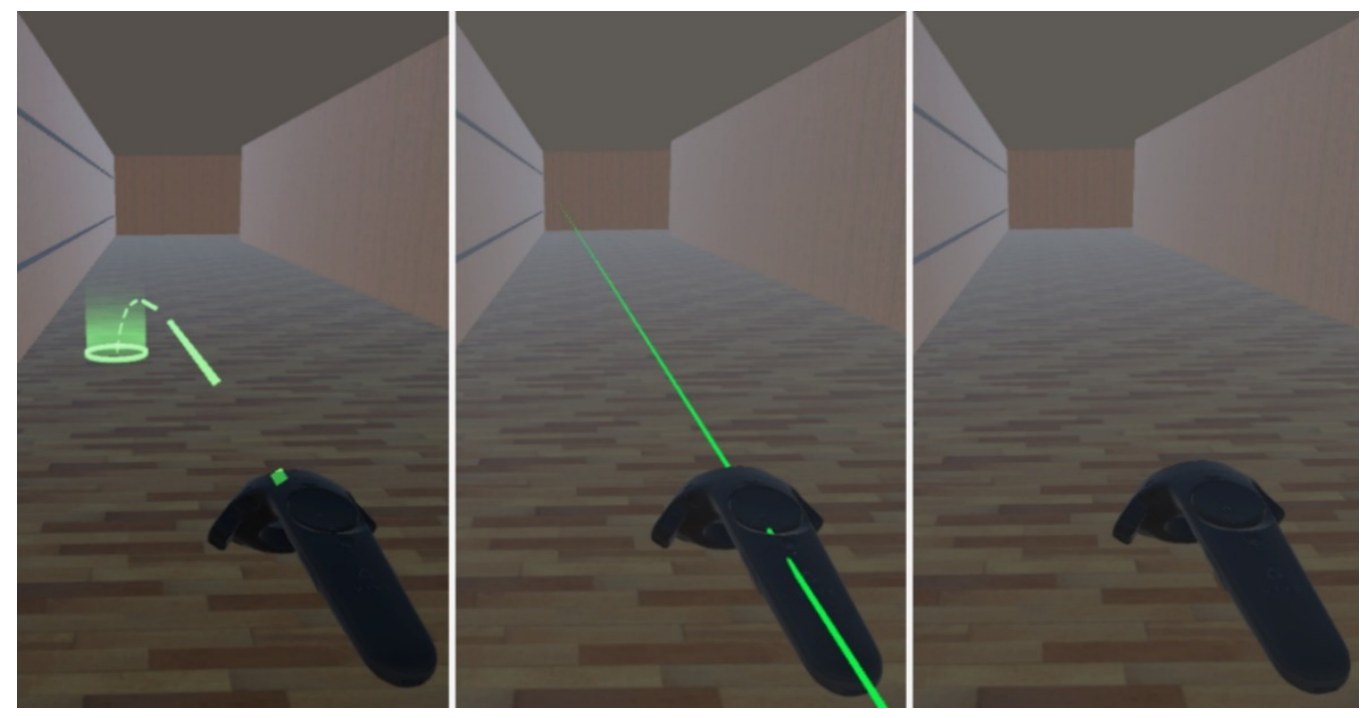

Figure 2. The locomotion techniques from left to right: teleport, slider, and grab.

\subsubsection{Grab}

Grab was motivated by the Go-Go technique [41], the locomotion technique used in Google Earth VR [43], and other techniques based on upper-body gestures such as Trigger Walking [44]. To travel with grab, the participant reached out with the controller, pressed down the touchpad, and moved the controller towards the body (see the right side of Figure 2). Grab was chosen for the current experiment because we hypothesized that the physical gesture of grabbing would provide motion cues for the vestibular and proprioceptive systems that are important in preventing simulator sickness [45].

The displacement of the controller $d_{c}$ was mapped to the transition distance $d_{t}$ in VR using a function

$$
d_{t}=m \times d_{c}
$$


where multiplier $m$ was set to 64 based on piloting. In practice, reaching further with the controller resulted in longer transition distances in VR.

\subsection{Procedure}

The experiment used a within-subject design where each participant evaluated all three techniques in a counterbalanced order. The experiment was divided into three blocks based on the three locomotion techniques.

At the beginning of each block, the current locomotion technique was introduced to the participants. They could rehearse the technique for a few minutes in the hall before proceeding to the experimental task. The task was to move in the corridor and count the number of grey dots on the left-side wall as accurately and quickly as possible. The experimental task was motivated by building information modeling applications where the user may need, for example, to search for deviations in physical structures. During the task, the participants could move freely backward and forward as well as closer and further from the wall. The participants were asked to press the trigger button of the controller before starting the task and again after finishing the task. These events were used for calculating task completion times. In each block, the task was carried out twice. The number of dots and their placement on the wall were different in the two tasks, but the total number of dots in each block (and with each technique) was always 34 .

After each block, the participants removed the VR headset and were asked to evaluate the used locomotion technique by filling in the SSQ and NASA-TLX. NASA-TLX was included because we were interested in understanding whether the techniques differed in the perceived workload. We used the unweighted NASA-TLX scoring procedure since it saves time and is as reliable as the weighted scoring procedure [46,47]. In addition, four 9-point scales ranging from -4 to +4 were used to assess how the techniques performed in the task. These scales are introduced in Table 1. Finally, after completing all three blocks, the participants ranked the three techniques based on their suitability to the task. Conducting the experiment took approximately $35 \mathrm{~min}$.

Table 1. Task-related spatial awareness evaluation scales.

\begin{tabular}{ccc}
\hline Scale Explanation & -4 & +4 \\
\hline Moving was ... & Difficult & Easy \\
Perceiving own location when moving was ... & Difficult & Easy \\
Searching target dots was ... & Difficult & Easy \\
Differentiating between target dots when counting them was .... & Difficult & Easy \\
\hline
\end{tabular}

\subsection{Data Analysis}

Distributions of collected data (i.e., task completion times, number of gestures, number of errors, SSQ scores, NASA-TLX scores, and task-related spatial awareness evaluations) were checked for normality with Shapiro-Wilk tests. The results showed that none of the data were normally distributed. Therefore, Friedman tests and Wilcoxon signed-rank tests were used for statistical analyses. A Bonferroni corrected $p$-value of $0.05 / 3=0.016$ was used as the significance threshold. In analyses, a gesture was defined as an event where the participant pressed down the controller's touchpad, moved to the desired location, and released the touchpad. The purpose of this analysis was to understand possible differences in task completion times. The number of errors was defined as the difference between the reported number of dots and the actual number of dots on the wall.

\section{Results}

\subsection{Task Completion Times}

For the average task completion times (see Figure 3), a Friedman test showed a statistically significant effect of locomotion technique $\left(X^{2}=16.8, p<0.001, W=0.466\right)$. The results of pairwise comparisons showed that completing the task with teleport took 
significantly longer than with slider $(M D=30.4, p=0.002, r=0.744)$ and grab $(M D=27.6$, $p=0.001, r=0.790)$.

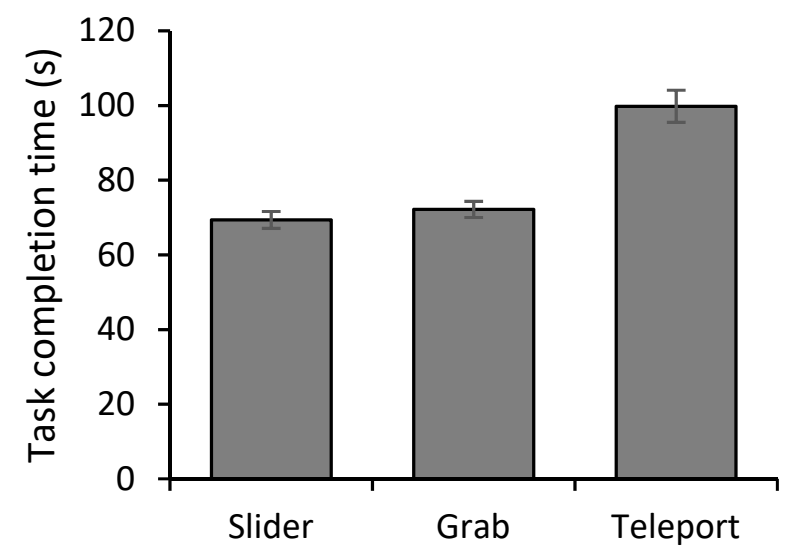

Figure 3. Average task completion times and standard errors of the mean (SEMs) for slider, grab, and teleport.

\subsection{Number of Gestures}

For the average number of gestures (see Figure 4), a Friedman test showed a statistically significant effect of locomotion technique $\left(X^{2}=11.4, p=0.003, W=0.318\right)$. The results of pairwise comparisons showed that completing the task with grab required significantly more gestures than slider $(M D=12.3, p=0.003, \mathrm{r}=0.693)$ and teleport $(M D=9.9, p=0.006$, $r=0.641)$.

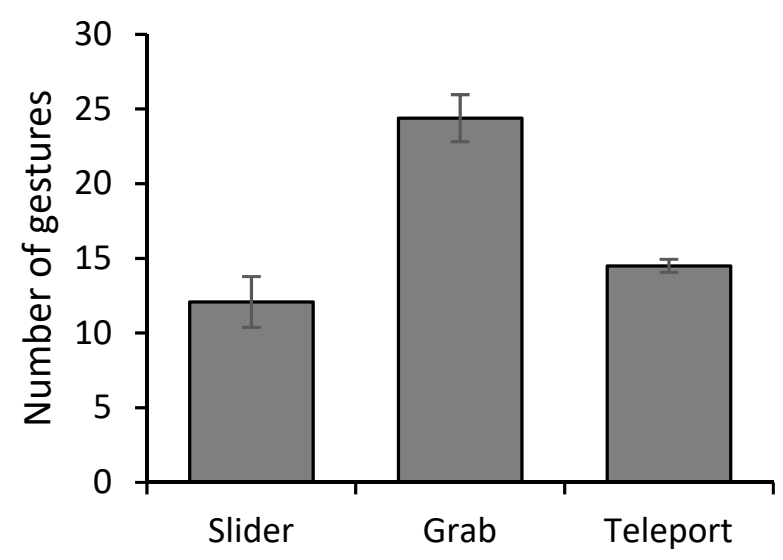

Figure 4. Average number of gestures and SEMs for slider, grab, and teleport.

\subsection{Number of Errors}

The result of a Friedman test showed no statistically significant effect of locomotion technique on the number of errors. The error distributions for each technique are shown in Figure 5. When using slider or grab, seven participants completed both tasks without errors. With teleport, three participants performed both tasks without errors. The number of errors per participant during the whole experiment ranged between 2 and 10 (average 4.4 , median 4). 

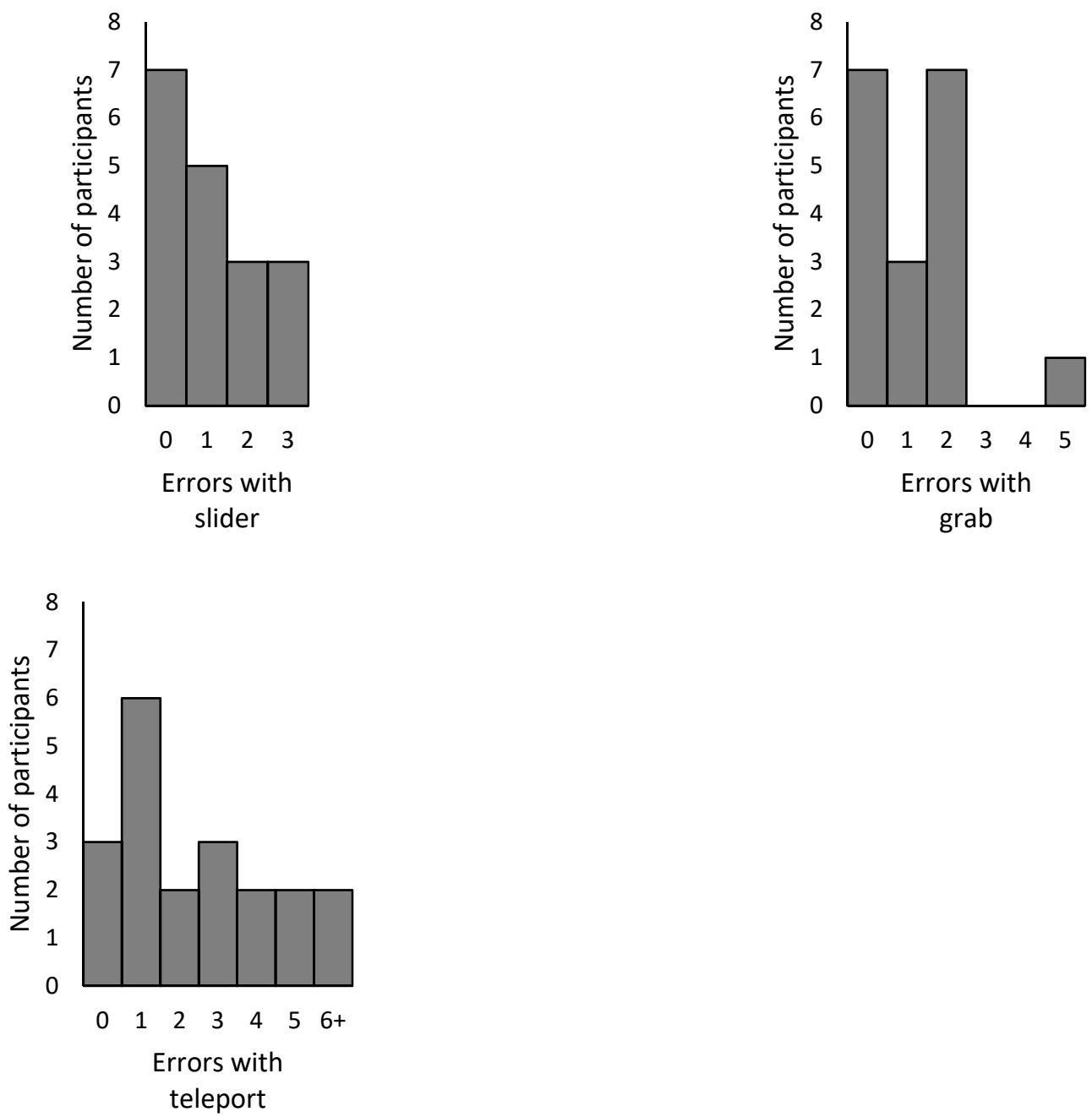

Figure 5. Error distributions for slider, grab, and teleport. Errors from both tasks are included.

\subsection{Simulator Sickness Questionnaire}

The results of four separate Friedman tests performed for SSQ total score, nausearelated subscore, oculomotor-related subscore, and disorientation-related subscore (see Figure 6) did not show statistically significant effects of locomotion technique.

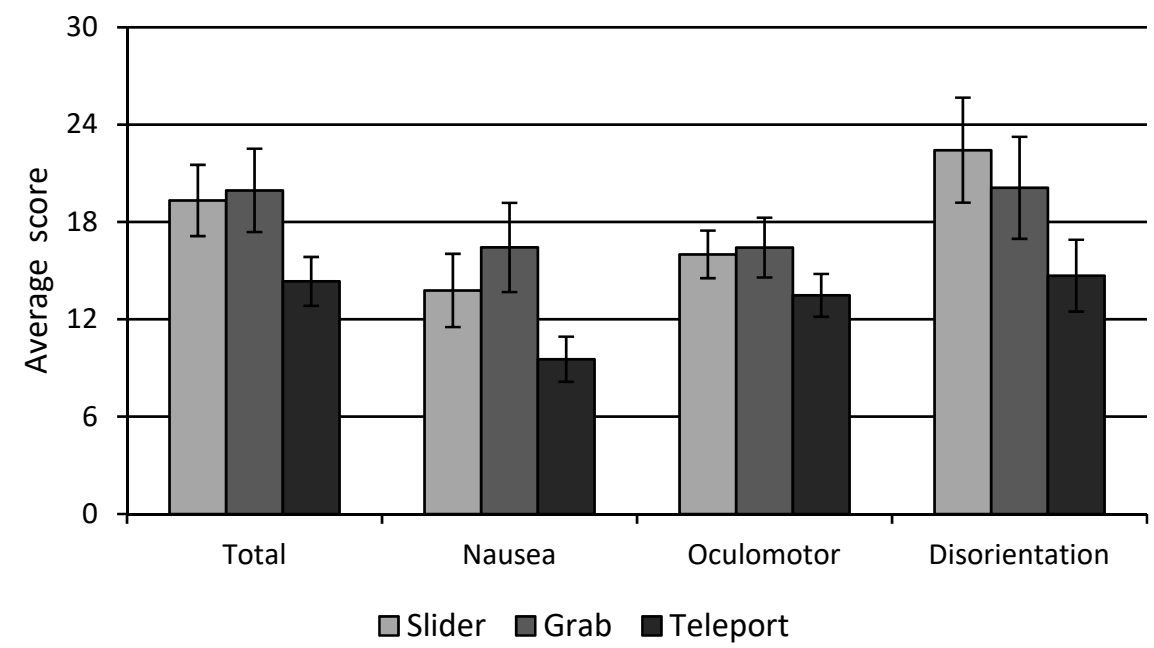

Figure 6. Average SSQ total score and subscores for each technique. Error bars represent SEMs. 


\subsection{NASA-TLX}

For the ratings of mental demand (see Figure 7), a Friedman test showed a statistically significant effect of locomotion technique $\left(X^{2}=14.6, p=0.001, W=0.830\right)$. The results of pairwise comparisons showed that participants rated the use of teleport as significantly more mentally demanding than slider $(M D=4.2, p=0.001, r=0.800)$ and grab $(M D=3.2$, $p=0.015, r=0.576$ ).

For the ratings of physical and temporal demand, the Friedman tests did not show statistically significant effects of locomotion technique.

For the ratings of performance, Friedman test showed a statistically significant effect of locomotion technique $\left(X^{2}=19.9, p<0.001, W=0.553\right)$. The results of pairwise comparisons showed that participants rated their performance with teleport as significantly worse than with slider $(M D=4.4, p<0.001, r=0.830)$ and grab $(M D=3.7, p=0.002, r=0.720)$.

For the ratings of effort, the Friedman tests showed a statistically significant effect of locomotion technique $\left(X^{2}=11.6, p=0.003, W=0.466\right)$. The results of pairwise comparisons showed that participants perceived teleport to require significantly more effort than grab $(M D=2.7, p=0.005, r=0.662)$.

For the ratings of frustration level, the Friedman tests showed a statistically significant effect of locomotion technique $\left(X^{2}=14.7, p=0.001, W=0.409\right)$. The results of pairwise comparisons showed that participants rated teleport as significantly more frustrating to use than slider $(M D=4.4, p=0.002, r=0.715)$ and grab $(M D=2.9, p=0.015, r=0.574)$.

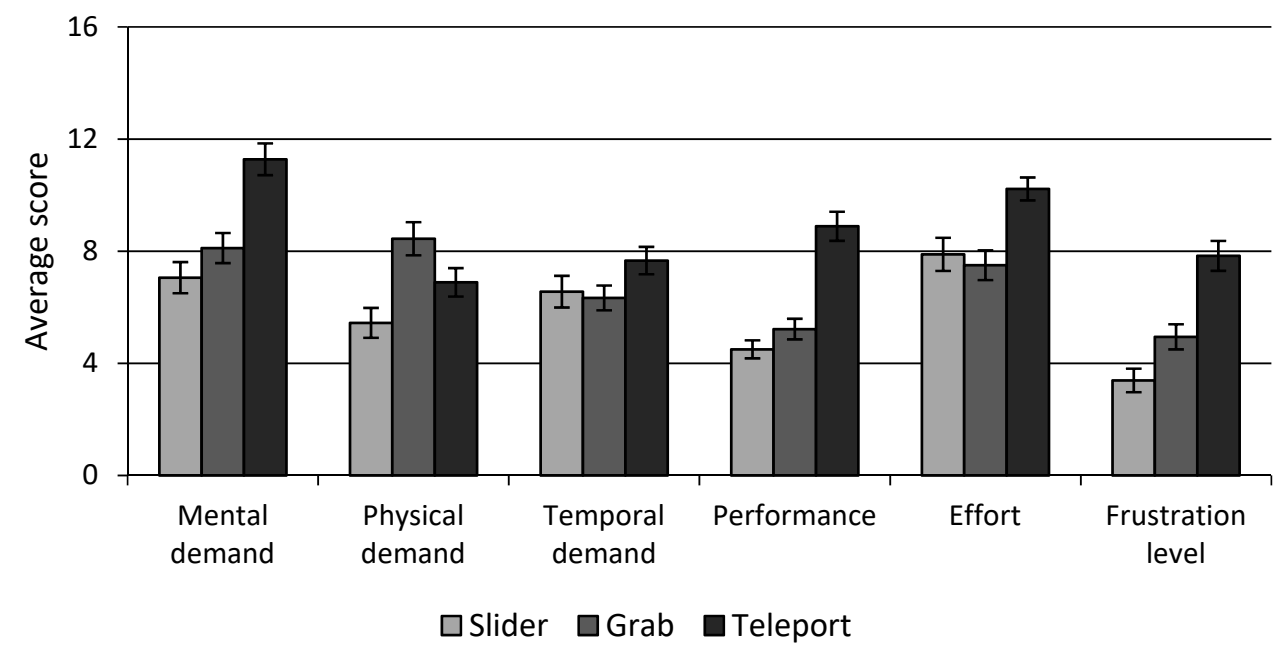

Figure 7. Average NASA-TLX ratings for each technique. Error bars represent SEMs.

\subsection{Task-Related Spatial Awareness Evaluation}

For the ratings of easiness of movement (see Figure 8), the Friedman tests did not show a statistically significant effect of the locomotion technique.

For the ratings of perceiving location, the Friedman tests showed a statistically significant effect of locomotion technique $\left(X^{2}=16.6, p<0.001, W=0.462\right)$. The results of pairwise comparisons showed that perceiving own location when moving was significantly more difficult with teleport than with slider $(M D=3.4, p=0.001, r=0.759)$ and grab $(M D=3.0$, $p=0.003, r=0.702$ ).

For the ratings of searching targets, the Friedman tests showed a statistically significant effect of locomotion technique $\left(X^{2}=19.8, p<0.001, W=0.551\right)$. The results of pairwise comparisons showed that searching targets was significantly more difficult with teleport than with slider $(M D=2.8, p=0.001, r=0.806)$ and grab $(M D=1.8, p=0.005, r=0.664)$.

For the ratings of differentiating targets, the Friedman tests showed a statistically significant effect of locomotion technique $\left(X^{2}=20.6, p<0.001, W=0.572\right)$. The results of pairwise comparisons showed that differentiating between targets was significantly more 
difficult with teleport than with slider $(M D=3.8, p<0.001, r=0.834)$ and grab $(M D=3.4$, $p=0.001, r=0.817)$.

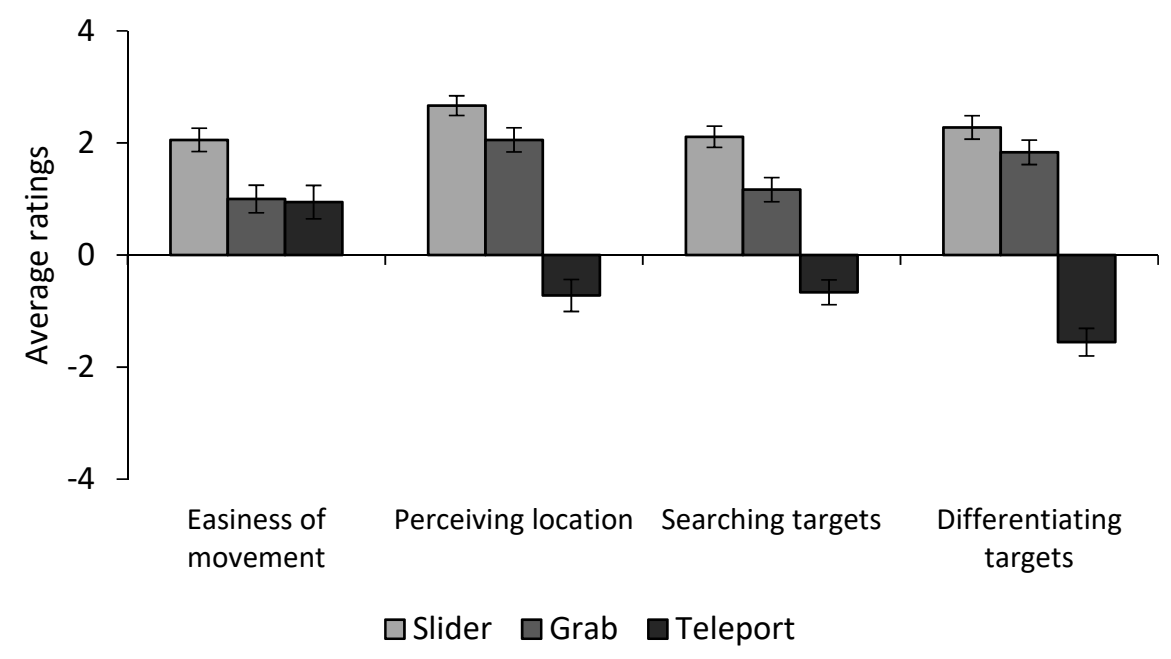

Figure 8. Average task-related ratings for each technique. Error bars represent SEMs.

\subsection{Preferences}

The participants ranked slider the most preferred technique, grab the second-most preferred technique, and teleport the least preferred technique for the experimental task (see Figure 9).
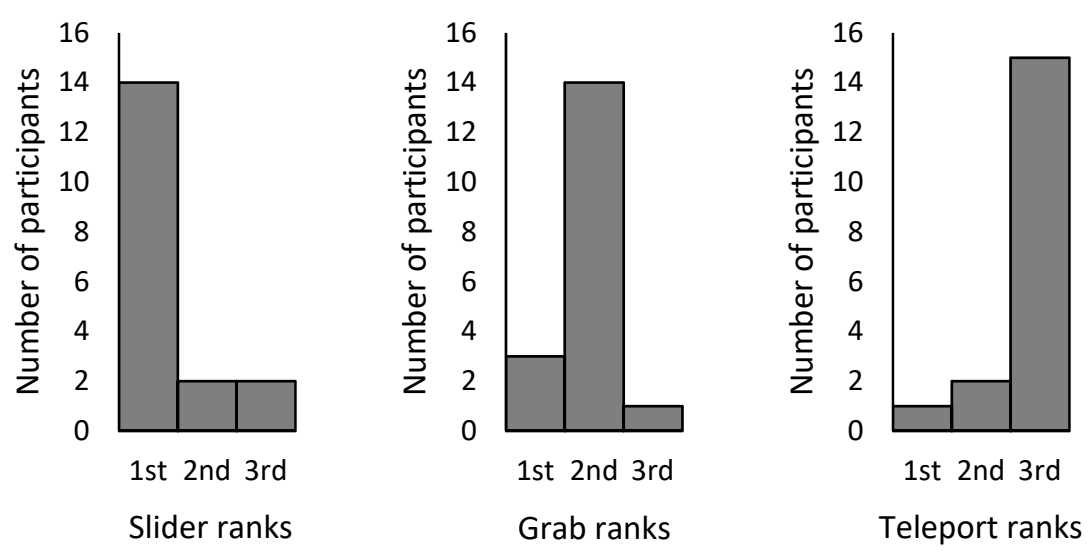

Figure 9. Ranking of the techniques from 1st (the most preferred) to 3rd (the least preferred).

\section{Discussion}

Our results showed that the two continuous locomotion techniques, slider and grab, were significantly faster to use than the non-continuous teleport in the current visual observation task. The SSQ scores for teleport tended to be lower than for slider and grab, but no statistically significant differences were found between the techniques. This could be due to the high variance in SSQ scores between participants.

The faster task completion times with slider and grab could be explained by looking at the results of the task-related ratings. The ratings indicated that it was significantly easier to perceive own location, search target dots, and differentiate between target dots with the continuous techniques. Thus, it seems that slider and grab were suited to the current task where spatial awareness was beneficial. On the contrary, when moving to a new location with teleport, it could be difficult to tell whether a dot on the wall had already been counted. This confusion and additional cognitive load may have resulted in longer task completion times with teleport. The cognitive load caused by teleport could also relate to the results of NASA-TLX ratings. The results showed that the use of teleport was perceived 
as more mentally demanding and frustrating compared to slider and grab. Additionally, the participants felt that teleport required more effort and led to worse performance. This subjective estimation of performance was in line with the measured task completion times.

Overall, the participants ranked slider as the most preferred technique for the current task. This could be due to the sliding metaphor that allowed participants to select the destination and travel with the desired velocity using a single gesture. The results indicated that completing the task with slider took significantly fewer gestures than with grab. It should be observed, however, that a single gesture was not sufficient because the participants used an average of 12 slider gestures for one task.

Our current work contributes to VR locomotion and HCI research in two ways. Firstly, we showed that it was possible to develop continuous, controller-based locomotion techniques that would not induce significantly more simulator sickness than teleport in a visual observation task. This is consistent with the results of a study where continuous movement with joystick did not cause more simulator sickness than teleport [14]. On the other hand, our findings differ from two studies where continuous movement initiated with a controller resulted in more simulator sickness than teleport $[29,35]$. Notable differences can be identified between our techniques and these two earlier studies. With slider, we used a line visualization to indicate the direction in which the participant started moving. This extra visualization may have helped reduce the mismatch between vision and the proprioceptive system because the participants initiated the movement with a physical gesture and could anticipate the direction of movement. Another factor possibly affecting the level of simulator sickness was related to how the participants perceived the visual movement induced by grab and slider. They could have either perceived themselves as being stable and the world in motion ("pulling the environment closer") or themselves in motion and the world being stable ("pulling myself in the environment"). The perception of "pulling the environment closer" could possibly reduce sensory conflict because the user is stationary both in the physical and virtual worlds. However, we did not ask the participants to report the perceived metaphor. Studying this is a potential topic for future research.

Secondly, our findings suggested that non-continuous teleport may not be ideal for a VR application where users focus on visual observation of a structure. There were limitations in the use of teleport in a task that required counting visual targets placed on a vertical surface. Even though the current environment was rather minimalistic, the results are in agreement with earlier work [30] where teleporting to new locations was correlated with increased user disorientation. However, the level of disorientation and spatial awareness resulting from the use of teleport may depend on the virtual environment. In an earlier study [29], where the task required spatial awareness, $75 \%$ of participants performed similarly with non-continuous teleport and continuous movement techniques. The study used an urban virtual environment with objects such as trees and cars, whereas the virtual wall structure used in our current study offered no other visual reference points than the grey dots. Thus, teleport as a locomotion technique may provide sufficient spatial awareness if there are enough visual reference points or boundaries [17] that can be utilized as anchors when moving to a new location.

One limitation of our current study was that the evaluated VR environment was rather simple because the experimental task required moving in a straight corridor. We plan to continue this research using a more complicated route with turns and obstacles. Furthermore, additional data should be collected with participants who have experience in building information modeling (e.g., architectural professionals). A larger sample size could also help in reducing variance in SSQ scores. Finally, for the sake of experimental research, the participants could use only one locomotion technique at a time. In practice, it would be more meaningful to allow users to utilize alternative locomotion techniques at will. Teleport is appropriate for quick travel between locations, whereas slider and grab are useful for moving at a slower pace when visual details are important during locomotion. A possible direction for future research would be to study more complex tasks that require 
utilizing complementary locomotion techniques optimized for either speed or accuracy. One example of such a complex task could be the design of natural resources and land use in a forestry planning context.

\section{Conclusions}

In summary, the work presented in this paper suggested that continuous VR locomotion techniques designed for stationary users do not necessarily induce significantly more simulator sickness than non-continuous teleport. Avoiding simulator sickness was one of the main reasons for choosing teleport as the default locomotion technique for VR experiences. However, considering the current findings, continuous locomotion techniques could be introduced at least as supplementary techniques to teleport, which is not an optimal technique for observing the environment during locomotion. Furthermore, the proposed techniques, slider and grab, were shown to be suited for the current task requiring spatial awareness. We see that these techniques could be applied to a range of multimodal VR applications. Examples include facility management and architectural design applications where structural details are essential, as well as tools for designing natural resources and land use in visually complex environments.

Author Contributions: Conceptualization, J.R., J.K., O.K., T.N. and R.R.; methodology, J.R., J.K. and T.N.; software, O.K., J.R. and J.K.; investigation, J.R. and J.K.; resources, R.R.; data curation, J.R.; writing—original draft preparation, J.R.; writing—review and editing, J.R., J.K., O.K., T.N. and R.R.; visualization, J.R. and J.K.; supervision, R.R.; project administration, R.R.; funding acquisition, R.R. All authors have read and agreed to the published version of the manuscript.

Funding: This research was funded by Business Finland (grant number 1444/31/2016) and the Academy of Finland (316804).

Institutional Review Board Statement: The study was conducted according to the guidelines of the Declaration of Helsinki.

Informed Consent Statement: Informed consent was obtained from all participants involved in the study.

Data Availability Statement: All data are contained within the manuscript. Raw data are available from the corresponding author upon request.

Conflicts of Interest: The authors declare no conflict of interest.

\section{References}

1. LaViola, J.J., Jr.; Kruijff, E.; Bowman, D.; Poupyrev, I.P.; McMahan, R.P. 3D User Interfaces: Theory and Practice, 2nd ed.; AddisonWesley: Boston, MA, USA, 2017.

2. Di Luca, M.; Seifi, H.; Egan, S.; Gonzalez-Franco, M. Locomotion Vault: The Extra Mile in Analyzing VR Locomotion Techniques. In Proceedings of the 2021 CHI Conference on Human Factors in Computing Systems, Yokohama, Japan, 8-13 May 2021; ACM: New York, NY, USA, 2021; pp. 1-10.

3. Boletsis, C. The New Era of Virtual Reality Locomotion: A Systematic Literature Review of Techniques and a Proposed Typology. Multimodal Technol. Interact. 2017, 1, 24. [CrossRef]

4. Wilson, P.T.; Kalescky, W.; MacLaughlin, A.; Williams, B. VR locomotion. In Proceedings of the 15th ACM SIGGRAPH Conference on Virtual-Reality Continuum and Its Applications in Industry-VRCAI '16, Zhuhai, China, 3-4 December 2016; ACM Press: New York, NY, USA, 2016; pp. 243-249.

5. Borrego, A.; Latorre, J.; Llorens, R.; Alcañiz, M.; Noé, E. Feasibility of a walking virtual reality system for rehabilitation: Objective and subjective parameters. J. Neuroeng. Rehabil. 2016, 13, 68. [CrossRef] [PubMed]

6. Sayyad, E.; Sra, M.; Hollerer, T. Walking and Teleportation in Wide-area Virtual Reality Experiences. In Proceedings of the 2020 IEEE International Symposium on Mixed and Augmented Reality (ISMAR), Porto de Galinhas, Brazil, 9-13 November 2020; pp. 608-617.

7. Bozgeyikli, E.; Raij, A.; Katkoori, S.; Dubey, R. Locomotion in Virtual Reality for Individuals with Autism Spectrum Disorder. In Proceedings of the 2016 Symposium on Spatial User Interaction-SUI '16, Tokyo, Japan, 15-16 October 2016; ACM Press: New York, NY, USA, 2016; pp. 33-42.

8. Harris, A.; Nguyen, K.; Wilson, P.T.; Jackoski, M.; Williams, B. Human joystick. In Proceedings of the 13th ACM SIGGRAPH International Conference on Virtual-Reality Continuum and Its Applications in Industry-VRCAI '14, Shenzhen, China, 30 November2 December 2014; ACM Press: New York, NY, USA, 2014; pp. 231-234. 
9. Hanson, S.; Paris, R.A.; Adams, H.A.; Bodenheimer, B. Improving Walking in Place Methods with Individualization and Deep Networks. In Proceedings of the 2019 IEEE Conference on Virtual Reality and 3D User Interfaces (VR), Osaka, Japan, 23-27 March 2019; pp. 367-376.

10. Grechkin, T.Y.; Plumert, J.M.; Kearney, J.K. Dynamic Affordances in Embodied Interactive Systems: The Role of Display and Mode of Locomotion. IEEE Trans. Vis. Comput. Graph. 2014, 20, 596-605. [CrossRef] [PubMed]

11. Fernandes, A.S.; Feiner, S.K. Combating VR sickness through subtle dynamic field-of-view modification. In Proceedings of the 2016 IEEE Symposium on 3D User Interfaces (3DUI), Greenville, SC, USA, 19-20 March 2016; pp. 201-210.

12. Keil, J.; Edler, D.; O’Meara, D.; Korte, A.; Dickmann, F. Effects of Virtual Reality Locomotion Techniques on Distance Estimations. ISPRS Int. J. Geo-Inf. 2021, 10, 150. [CrossRef]

13. Cardoso, J.C.S. Comparison of gesture, gamepad, and gaze-based locomotion for VR worlds. In Proceedings of the 22nd ACM Conference on Virtual Reality Software and Technology_VRST '16, Munich, Germany, 2-4 November 2016; ACM Press: New York, NY, USA, 2016; pp. 319-320.

14. Bozgeyikli, E.; Raij, A.; Katkoori, S.; Dubey, R. Point \& Teleport Locomotion Technique for Virtual Reality. In Proceedings of the 2016 Annual Symposium on Computer-Human Interaction in Play-CHI PLAY '16, Austin, TX, USA, 16-19 October 2016; ACM Press: New York, NY, USA, 2016; pp. 205-216.

15. Schäfer, A.; Reis, G.; Stricker, D. Controlling Teleportation-Based Locomotion in Virtual Reality with Hand Gestures: A Comparative Evaluation of Two-Handed and One-Handed Techniques. Electronics 2021, 10, 715. [CrossRef]

16. Habgood, J.; Moore, D.; Wilson, D.; Alapont, S. Rapid, Continuous Movement between Nodes as an Accessible Virtual Reality Locomotion Technique. In Proceedings of the 2018 IEEE Conference on Virtual Reality and 3D User Interfaces (VR), Tuebingen/Reutlingen, Germany, 18-22 March 2018; pp. 371-378.

17. Cherep, L.A.; Lim, A.F.; Kelly, J.W.; Acharya, D.; Velasco, A.; Bustamante, E.; Ostrander, A.G.; Gilbert, S.B. Spatial cognitive implications of teleporting through virtual environments. J. Exp. Psychol. Appl. 2020, 26, 480-492. [CrossRef] [PubMed]

18. Clifton, J.; Palmisano, S. Effects of steering locomotion and teleporting on cybersickness and presence in HMD-based virtual reality. Virtual Real. 2020, 24, 453-468. [CrossRef]

19. Kelly, J.W.; Ostrander, A.G.; Lim, A.F.; Cherep, L.A.; Gilbert, S.B. Teleporting through virtual environments: Effects of path scale and environment scale on spatial updating. IEEE Trans. Vis. Comput. Graph. 2020, 26, 1841-1850. [CrossRef] [PubMed]

20. Nescher, T.; Huang, Y.-Y.; Kunz, A. Planning redirection techniques for optimal free walking experience using model predictive control. In Proceedings of the 2014 IEEE Symposium on 3D User Interfaces (3DUI), Minneapolis, MN, USA, 29-30 March 2014; pp. 111-118.

21. Bruder, G.; Lubos, P.; Steinicke, F. Cognitive Resource Demands of Redirected Walking. IEEE Trans. Vis. Comput. Graph. 2015, 21, 539-544. [CrossRef] [PubMed]

22. Nguyen, L.T.N.; Eager, D.; Nguyen, H.T. Effect of Wearing Whole Body Compression Garments on Cardiovascular Function using ECG Signals. In Proceedings of the Telehealth and Assistive Technology/847: Intelligent Systems and Robotics, Zurich, Switzerland, 6-8 October 2016; ACTA Press: Calgary, AB, Canada, 2016; pp. 23-29.

23. Nilsson, N.C.; Peck, T.; Bruder, G.; Hodgson, E.; Serafin, S.; Whitton, M.; Steinicke, F.; Rosenberg, E.S. 15 Years of Research on Redirected Walking in Immersive Virtual Environments. IEEE Comput. Graph. Appl. 2018, 38, 44-56. [CrossRef] [PubMed]

24. McCullough, M.; Xu, H.; Michelson, J.; Jackoski, M.; Pease, W.; Cobb, W.; Kalescky, W.; Ladd, J.; Williams, B. Myo arm. In Proceedings of the ACM SIGGRAPH Symposium on Applied Perception-SAP '15, Tübingen, Germany, 13-14 September 2015; ACM Press: New York, NY, USA, 2015; pp. 107-113.

25. Paris, R.A.; McNamara, T.P.; Rieser, J.J.; Bodenheimer, B. A comparison of methods for navigation and wayfinding in large virtual environments using walking. In Proceedings of the IEEE Virtual Reality, Los Angeles, CA, USA, 18-22 March 2017; pp. 261-262.

26. Tregillus, S.; Folmer, E. VR-STEP: Walking-in-Place using Inertial Sensing for Hands Free Navigation in Mobile VR Environments. In Proceedings of the 2016 CHI Conference on Human Factors in Computing Systems-CHI '16, San Jose, CA, USA, 7-12 May 2016; ACM Press: New York, NY, USA, 2016; pp. 1250-1255.

27. Kitson, A.; Hashemian, A.M.; Stepanova, E.R.; Kruijff, E.; Riecke, B.E. Comparing leaning-based motion cueing interfaces for virtual reality locomotion. In Proceedings of the 2017 IEEE Symposium on 3D User Interfaces (3DUI), Los Angeles, CA, USA, 18-19 March 2017; pp. 73-82.

28. Kruijff, E.; Marquardt, A.; Trepkowski, C.; Lindeman, R.W.; Hinkenjann, A.; Maiero, J.; Riecke, B.E. On Your Feet! Enhancing Vection in Leaning-Based Interfaces through Multisensory Stimuli. In Proceedings of the 2016 Symposium on Spatial User Interaction-SUI '16, Tokyo, Japan, 15-16 October 2016; ACM Press: New York, NY, USA, 2016; pp. 149-158.

29. Weibker, T.; Kunert, A.; Frohlich, B.; Kulik, A. Spatial Updating and Simulator Sickness During Steering and Jumping in Immersive Virtual Environments. In Proceedings of the 2018 IEEE Conference on Virtual Reality and 3D User Interfaces (VR), Tuebingen/Reutlingen, Germany, 18-22 March 2018; pp. 97-104.

30. Bowman, D.A.; Koller, D.; Hodges, L.F. Travel in immersive virtual environments: An evaluation of viewpoint motion control techniques. In Proceedings of the IEEE 1997 Annual International Symposium on Virtual Reality, Albuquerque, NM, USA, 1-5 March 1997; pp. 45-52.

31. Paris, R.; Klag, J.; Rajan, P.; Buck, L.; McNamara, T.P.; Bodenheimer, B. How Video Game Locomotion Methods Affect Navigation in Virtual Environments. In Proceedings of the ACM Symposium on Applied Perception 2019, Barcelona, Spain, 19-20 September 2019; ACM: New York, NY, USA, 2019; pp. 1-7. 
32. Bhandari, J.; MacNeilage, P.; Folmer, E. Teleportation without spatial disorientation using optical flow cues. In Proceedings of the Graphics Interface, Toronto, ON, Canada, 8-11 May 2018; pp. 153-158.

33. Bolte, B.; Bruder, G.; Steinicke, F. The Jumper Metaphor: An Effective Navigation Technique for Immersive Display Setups. In Proceedings of the Virtual Reality International Conference (VRIC), Laval, France, 6-8 April 2011; pp. 1-7.

34. Langbehn, E.; Lubos, P.; Steinicke, F. Evaluation of Locomotion Techniques for Room-Scale VR. In Proceedings of the Virtual Reality International Conference-Laval Virtual on-VRIC '18, Laval, France, 4-6 April 2018; ACM Press: New York, NY, USA, 2018; pp. 1-9.

35. Frommel, J.; Sonntag, S.; Weber, M. Effects of controller-based locomotion on player experience in a virtual reality exploration game. In Proceedings of the International Conference on the Foundations of Digital Games_FDG '17, Hyannis, MA, USA, 14-17 August 2017; ACM Press: New York, NY, USA, 2017; pp. 1-6.

36. Cao, Z.; Jerald, J.; Kopper, R. Visually-Induced Motion Sickness Reduction via Static and Dynamic Rest Frames. In Proceedings of the 2018 IEEE Conference on Virtual Reality and 3D User Interfaces (VR), Tuebingen/Reutlingen, Germany, 18-22 March 2018; pp. 105-112.

37. Waller, D.; Hodgson, E. Sensory Contributions to Spatial Knowledge of Real and Virtual Environments. In Human Walking in Virtual Environments; Steinicke, F., Visell, Y., Campos, J., Lécuyer, A., Eds.; Springer: New York, NY, USA, 2013; pp. 3-26. ISBN 978-1-4419-8431-9.

38. Kennedy, R.S.; Lane, N.E.; Berbaum, K.S.; Lilienthal, M.G. Simulator Sickness Questionnaire: An Enhanced Method for Quantifying Simulator Sickness. Int. J. Aviat. Psychol. 1993, 3, 203-220. [CrossRef]

39. Hart, S.G.; Staveland, L.E. Development of NASA-TLX (Task Load Index): Results of Empirical and Theoretical Research. In Advances in Psychology; Hancock, P.A., Meshkati, N., Eds.; Human Mental Workload; Elsevier: Amsterdam, The Netherland, 1988; Volume 52, pp. 139-183.

40. Valve SteamVR. Available online: https:/ / steamcommunity.com/steamvr (accessed on 26 March 2021).

41. Bowman, D.A.; Johnson, D.B.; Hodges, L.F. Testbed evaluation of virtual environment interaction techniques. In Proceedings of the ACM Symposium on Virtual Reality Software and Technology—VRST ‘99, London, UK, 20-22 December 1999; ACM Press: New York, NY, USA, 1999; pp. 26-33.

42. Rahimi Moghadam, K.; Banigan, C.; Ragan, E.D. Scene Transitions and Teleportation in Virtual Reality and the Implications for Spatial Awareness and Sickness. IEEE Trans. Vis. Comput. Graph. 2018, 26, 2273-2287. [CrossRef] [PubMed]

43. Google Google Earth. Available online: https:/ / arvr.google.com/earth/ (accessed on 26 March 2021).

44. Sarupuri, B.; Chipana, M.L.; Lindeman, R.W. Trigger Walking: A low-fatigue travel technique for immersive virtual reality. In Proceedings of the 2017 IEEE Symposium on 3D User Interfaces (3DUI), Los Angeles, CA, USA, 18-19 March 2017; pp. 227-228.

45. Davis, S.; Nesbitt, K.; Nalivaiko, E. A Systematic Review of Cybersickness. In Proceedings of the 2014 Conference on Interactive Entertainment, Newcastle, NSW, Australia, 2-3 December 2014; ACM: New York, NY, USA, 2014; Volume 19, pp. 1-9.

46. Wiebe, E.N.; Roberts, E.; Behrend, T.S. An examination of two mental workload measurement approaches to understanding multimedia learning. Comput. Hum. Behav. 2010, 26, 474-481. [CrossRef]

47. Moroney, W.F.; Biers, D.W.; Eggemeier, F.T.; Mitchell, J.A. A comparison of two scoring procedures with the NASA task load index in a simulated flight task. In Proceedings of the IEEE 1992 National Aerospace and Electronics Conference@m_NAECON 1992, Dayton, OH, USA, 18-22 May 1992; pp. 734-740. 